\title{
Tratamiento esteroidal en distrofia muscular de Duchenne
}

\author{
Drs. Camen Luz Aviles L., Carmen Gutiérrez S., ${ }^{1}$ Fernando Novou S., \\ Sra. Eugenia Gil A., ${ }^{3}$ Dr. Andrés Stuardo L., 4
}

Corticosteroids in the treatment of Duchenne's Muscular dystrophy

\begin{abstract}
Eigh patients with Duchenne's Muscolar Distraphy, 5 to 11 years old, were diagnosed by the clinical picture, muscular biopsy, electromiography (EMC) and CPK. All were able to walk and recejved Prednisone $3 \mathrm{mg} / \mathrm{kg}$ weight every other day for one year in the Outpatient Clinic. They were compared with a group of patients with the ame chanacteristics but without treatment.

Follow-up included evaluation of muscular strenght, physical activity electromiography, and CPK leyels.

Although clinical improvement was observed at the onset of corticosteroidal treatment, the clinical course was not different from those not treated. CPK levels showed a wide variation and no comelation was found with the clinical course. EMG remained unchanged at one year follow-up.
\end{abstract}

La Distrofia Muscular Pseudohipertrófica de Duchenne (DMD) es una miopatia progresiva, de herencia recesiva ligada al sexo, que comienza durante la nin̄ez temprana, comprometiendo primariamente los músculos de las cinturas pelviana y escapular, para luego generalizarse, llevando a la invalidez entre los 10 y 14 años de edad.' La muerte sobreviene antes de los 20 años en la gran mayoria de los casos. Generalmente la capacidad Intelectual permanece indemne y el niño asiste a su progresiva limitación.

Desde su dẹscripción por Duchenne, el año 1868, poco se ha agregado a la descripción clínica de esta enfermedad..$^{2}$ Aunque los métodos diagnósticos han sido considerablemente refinados, su causa es desconocida y aún no se dispone de un tratamiento que detenga su curso irreversible.

En investigaciones realizadas en la década del 60 , se observaron resultados contradictorios con el uso de corticoides en el tratamiento de ella ${ }^{3,4,5}$

Basados en una experiencia del año 1974, que

\footnotetext{
IBecados en Pediatria, Hospital Paula Jaraquemada.

2Jefe del Servicio de Neurosiquiatria, Hospital Pauia Jaraquemada.

${ }^{3}$ Kinesiólogga, Departamento Medicina Física y Rehabilitación, Hospital Paula Jaraquemada.

4 Jefe del Departamento de Neurofisiologí. Hospitul del Salvador.
}

sugeria que la Prednisona era útil como tratamiento paliativo temporal para algunos pacientes con $\mathrm{DMD}^{6}$, decidimos abservar sus efectos en una serie de pacientes controlados en nuestra Únidad.

\section{MATERIAL Y METODO}

Se selecciono un gmupo de 8 Pacientes con DMD, diagnosticados por el cuadro clínico, biopsia muscular (Fig. 1), Electromiografía (EMG) y Creatinfosfokinasa (CPK), que no habian perdido la deambulación y cuyas edades fluctuaban entre 5 años 1/12 y 11 años 5/12. Se realizó examen físico general y estudios complementarios que incluían Radiografía de Tórax, PPD, evaluación de fuerza muscular y actividad física. Este grupo fue tratado con Prednisona (Laboratorio Chile), en dosis de 3 mg. por kilo de peso en días alternos, durante un período de un año. En todos los casos se obtuvo el consentimiento de los padres antes de iniciar el tratamiento.

El seguimiento se realizó con medición de fuerza muscular, actividad fisica, niveles de CPK y EMG. La fuerza muscular se evaluó según el método de KENDALL. ${ }^{7}$ Se comparan fuerzas musculares mediante el "Score" Muscular Total," que representa un promedio aritmético de la fuerza muscular en los 43 músculos evaluados. La actividad física se midió según los siguientes 


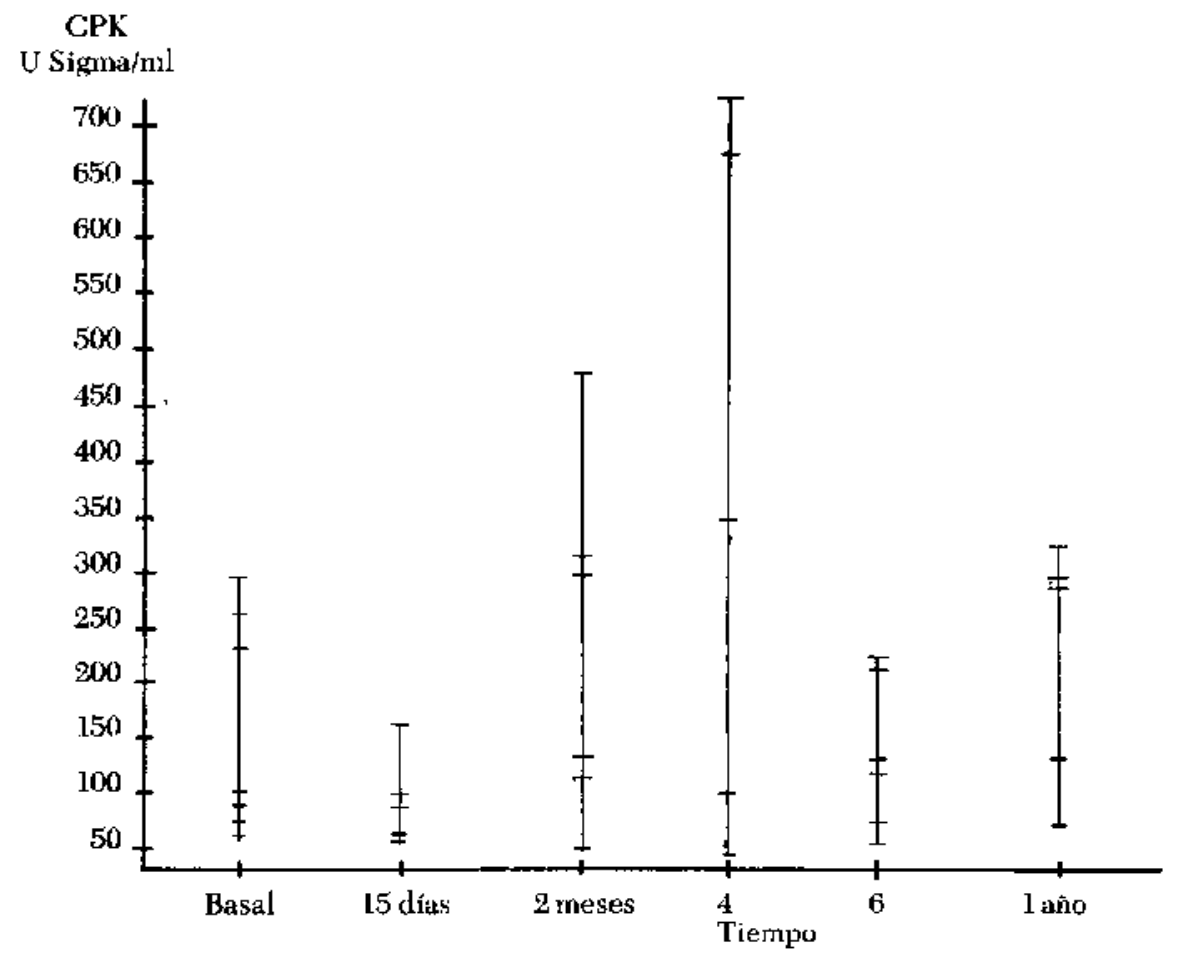

parámetros: Tiempo de Marcha, capacidad para ponerse de pie desde decúbito y sentado, correr, saltar en ambos y un pie y subir escalas que se calificaron en una escala de 0 a 28 puntos, otorgándose 4 puntos a cada actividad realizada sin dificultad. Todas las evaluaciones fueron realizadas por el mismo examinador mensualmente hasta los 6 meses y luego cada 2 meses. Los niveles séricos de CPK de midieron por el método colorimétrico $\mathrm{N}^{0} 661$ de Sigmal4 al comienzo, 15 días, $2,4,6$, y 12 meses de tratamiento. El contro] EMG. se realizó a $\operatorname{los} 3,6$ y $\mathbf{1 2}$ meses con electromiógrafo TECA TE4.

Los pacientes se controlaron periódicamente con el propósito dé pesquisar efectos colaterales del tratamiento esteroidal. Concomitantemente se administró Isoniazida a dosis de $10 \mathrm{mg}$ por kilo por día durante todo el período de cratamiento esteroidal.

Se comparan los valores obtenidos en las distintas evaluaciones en el grupo tratado.
Al finalizar el tratamiento se evaluó con los mismos parámetros de actividad fisica y fuerza muscular a un grupo de 7 nin̄os de edades similares, portadores de DMD que no habían recibido tratamiento, comparándose ambos grupos.

\section{RESULTADOS}

En la Tabla 1 se esquematizan los resultados del "Score" Muscular Total (SMT) obtenidos en los 8 casos tratados al inicio, 6 meses y un año de tratamiento.

Encontramos diferencias en el estado clínico de los pacientes independiente de la edad, así el paciente más dañado fue el N. ${ }^{\circ} 6$ y el menos, el N. ${ }^{\circ} 1$, siendo sus edades similares.

Se observó una mejoria de la fuerza muscular en todos los casos a los 6 meses de tratamiento, valores estadísticamente significativos $(P<0,01)$, la que se mantuvo al año de tratamiento en 7 de los 8 
Tabla 1

Evaluación de fuerza ruscular por Score Muscular Total (SMTị en los pacientes con tratamiento esteroidal

\begin{tabular}{ccccc}
\hline Caso & Edad (años/meses) & SMT inicial & SMT 6 meses & SMT 12 meses \\
\hline 1 & $10 / 5$ & 63 & 75.5 & 74.6 \\
2 & $11 / 5$ & 46.4 & 55.3 & 53.1 \\
3 & $6 / 2$ & 59.3 & 67.6 & 67.6 \\
4 & $5 / 4$ & 57 & 66 & 66 \\
5 & $5 / 8$ & 63.2 & 69.5 & 69.5 \\
6 & $10 / 7$ & 49.7 & 50.5 & 58.2 \\
7 & $9 / 2$ & 57 & 59.7 & 58.4 \\
8 & $5 / 1$ & 59 & 62.4 & 61.4 \\
\hline $\mathrm{X}$ & & 56.83 & 63.3 & $<0.1$ \\
\hline $\mathrm{P}$ & & & $<0.01$ &
\end{tabular}

Tabla 2

Evaluación de actividad física en los pacientes con tratamiento esteroidal

\begin{tabular}{cccc}
\hline Caso & Inicial & 6 meses & l año \\
\hline 1 & 27 & 28 & 25 \\
2 & 6 & 8 & 3 \\
3 & 18 & 23 & 26 \\
4 & 13 & 19 & 19 \\
5 & 22 & 27 & 27 \\
6 & 4 & 6 & 0 \\
7 & 9 & 15 & 15 \\
8 & 11 & 16 & 16 \\
\hline $\mathrm{X}$ & 13.75 & 17.75 & 16.38 \\
\hline $\mathrm{P}$ & & $<0.001$ & $<0.2$ \\
\hline
\end{tabular}

pacientes, a un nivel más bajo, sin ser significativa con respecto al estado inicial $(P<0,1)$.

El paciente $N .^{\circ} 6$ mostró deterioro de su fuerza muscular desde los 7 meses de tratamiento, perdiendo además la capacidad de deambulación, motivo por el cual se suspendió gradualmente el tratamiento esteroidal.

Los resultados en las evaluaciones de activjdad física se esquematizan en la Tabla 2 , observándose mejoria en todos los niños a los 6 meses de tratamiento, estadísticamente significativa ( $P<0,001$ ), la que se mantiene solamente en 5 pacientes al año de tratamiento $(P<0,2)$.

En la Tabla 3 observamos los valores de SMT y actividad fisica en los niños tratados después de un año de tratamiento, comparados con los valores

Tabla 3

Comparación de SMT y actividad física en niños tratados 1 año con Prednisona y niños que no recibieron tratamiento

\begin{tabular}{|c|c|c|c|c|}
\hline \multirow[t]{2}{*}{ Caso } & \multicolumn{2}{|c|}{ SMT } & \multicolumn{2}{|c|}{ Actividad tisica } \\
\hline & $\begin{array}{c}\text { Tratados } \\
\bar{x} \text { edad } 7.8 \text { años }\end{array}$ & $\begin{array}{l}\text { Sin tratamiento } \\
\bar{x} \text { edad } 8.1 \text { años }\end{array}$ & Tratado & Sin tratamiento \\
\hline I & 74.6 & 40.2 & 25 & 3 \\
\hline 2 & 53.1 & 42 & 3 & 1 \\
\hline 3 & 67.6 & 55.3 & 26 & 12 \\
\hline 4 & 66 & 62.3 & 19 & 16 \\
\hline 5 & 69.5 & 53.6 & 27 & 19 \\
\hline 6 & 43.2 & 28.9 & 0 & 0 \\
\hline 7 & 58.8 & 67 & 15 & 28 \\
\hline 8 & 58.4 & & 16 & \\
\hline $\mathbf{x}$ & 61.4 & 50.13 & 16.38 & 1129 \\
\hline $\mathbf{P}$ & & & & \\
\hline
\end{tabular}

$\overline{\mathrm{X}}=$ promedio 
en los niños que no lo recibieron, no observándose diferencias significativas entre ambos grupos ( $\mathrm{P}<0,02$ y $\mathrm{P}>0,2)$.

El análisis de los niveles de CPK en el grupo tratado mostró gran variabilidad, falta de correlación con el estado clínico de los pacientes y ausencia de diferencias significativas con respecto a los valores iniciales (Grafico 1). Tampoco observamos diferencias significativas en los niveles de CPK entre los niños tratados durante un año y los que no recibieron tratamiento esteroidal (Gráfico 2).

La EMG realizada en los músculos cuádriceps, gemelo interno, tibial anterior y glúteo medio, mostró potenciales de bajo voltaje, polifásicos, con espigas finas y trazado diferencial ante mínjmos esfuerzos, que representan caracteres específicos de distrofia muscular, destacando además la

\section{Gráfico 2}

Niveles de CPK en niños tratados (l año) en relación a niños sin tratamiento

\section{CPK}

U Sigma/m]

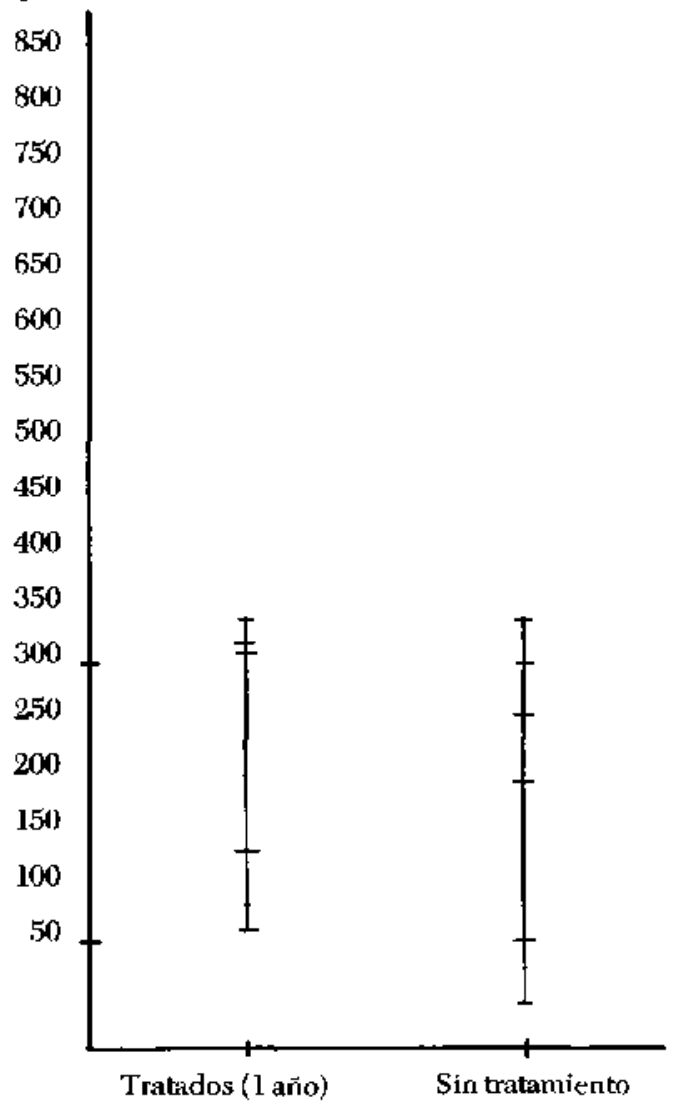

presencia de fibrilación en reposo, ondas agudas positivas en reposo y descargas bizarmas en inserción (ráfagas miotónicas y descargas de alta frecuencia), hallazgos más característicos de DMD.

Los controles EMG no mostraron variaciones durante el tratamiento esteroidal.

Todos los pacientes tratados mostraron la facie característica del Síndrome de Cushing y ligera ganancia de peso, llegando a la obesidad 2 de ellos. Hubo alteraciones de la conducta con hiperactivịdad en 3 casos. No se observó elevación de la presión arterial, ni otros efectos secundarios al tratamiento esteroidal.

\section{DISCUSION}

Se han realizado múltiples experiencias con distintos esquemas terapéuticos en el tratamiento de la DMD, sin lograrse hasta ahora un resultado efectivo en detener su curso irremediablemente progresivo. 8

El deterioro lineal y continuo de la fuerza muscular con la edad es una característica fundamental en la DMD. La estabilización o mejoria de las fuerzas aun por periodos cortos parece no ocurrir. Aunque la debilidad aumenta inexorablemente con la edad, la velocidad a la cual esto sucede varía de un paciente a otro, resultando en un amplio espectro de severidad cuando se comparan enfermos de edades similares, ${ }^{9}$ lo que dificulta la evaluación de la eficacia de un tratamiento dado.

Nuestros resultados muestran una transitoria mejoría en fuerza muscular y actividad física después de un período de tratamiento de 6 meses con Prednisona a días altemos, con valores estadísticamente significativos. Sin embargo, la enfermedad sigue su curso progresivo aun en los pacientes con tratamiento esteroidal, que al cabo de un año no mostraban mejoría significativa en ambos parámetros respecto al estado inicial $\mathrm{e}$ incluso un paciente perdió la deambulación.

El estado clínico de nuestros pacientes al término de un año de tratamiento, en relación a un gnupo de niños de edades similares que no lo habían recibido, no fue significativamente mejor. Estos resultados son discutibles dado el número de pacientes y el corto periodo de seguimiento, pero son concordantes con los de Siegel y col., que encontraron un transitorio y mínimo enlentecimiento del deterioro en pacientes tratados por un período de 3 años con Prednisolona, continuando 
la enfermedad su curso progresivo, a pesar del mismo. ${ }^{10}$

Los niveles séricos de CPK proporcionan un indicador útil de la presencia y grado de actividad de muchos desórdenes neuromusculares. " En general, altos niveles indican una amplia mionecrosis y la elevación de los niveles sugiere progresión de la enfermedad. Lo inverso, sin embargo, no es necesariamente verdadero, así la caída de los niveles de CPK se ve en niños con DMD después de los 10 años, aun cuando la enfermedad continúa progresando. Esta disminución puede ser atribuida a reducción de las reservas de CPK, pérdida de las fibras musculares, confinamiento en silla de ruedas, inactividad o cambios hormonales puberales.

Los efectos de los corticoides sobre los niveles séricos de CPK en DMD han sido contradictorios, observándose caída 0 aumento de ellos según distintos autores, ${ }^{12,13}$ sin correlación con la respuesta clínica. Nuestros valores fueron fluctuantes $y$ no mostraron una clara tendencia en ninguno de los sentidos.

\section{CONCLUSION}

Aunque la Prednisona puede provocar una mejoría transitoria inicial en pacientes con Distrofia Muscular de Duchenne (DMD), nuestros resultados le niegan valor terapéutico en detener el curso irreversible de esta enfermedad.

\section{RESUMEN}

Ocho pacientes con Distrofia Muscular de Duchenne, diagnosticados por las manifestaciones clinicas, biopsia muscular, creatinfosfokinasa (CPK) y electromiografía, de edades entre 5 años $1 / 12$ y 11 años 5/12, fueron tratados con Prednisona (Laboratorio Chile) $3 \mathrm{mg}$ por $\mathrm{Kg}$ de peso, en dias altemos, durante un período de un año.

Los pacientes no habían perdido la deambulación. El seguimiento se realizó con medición de fuerza muscular, actividad física, niveles de CPK y electromielografía.

Este grupo de enfermos se compara al término del tratamiento con un grupo de nifios portadores de DMD de edades similares que no recibieron tratamiento esteroidal.

Los resultados muestran una significativa mejoría $(P<0,010)$, en fuerza muscular (medido por Score Muscular Total) y actividad fisica ( $\mathbf{P}<$
$0,001)$ a los 6 meses de tratamiento. Al año de tratamiento no hay diferencia significativa en ambos parámetros con respecto al estado inicial. No se obseryaron diferencias en fuerza muscular $y$ actividad física al año de tratamiento con respecto al grupo sin tratamiento.

Los valores de CPK no mostraron diferencias significutivas respecto a los valores iniciales.

La electromiografía no mostró variaciones significativas en el curso del tratamiento.

Aunque el número de pacientes es reducido, nuestra experiencia muestra que los corticoides no tienen efectos benéficos en la evolución clínica de la DMD.

\section{RECONOCIMIENTO}

Las CPK se realizaron en la Unidad de Neuropsicología del Instituto de Nutrición y Tecnología de los Alimentos. Universidad de Chile.

Agradecemos también al Laboratorio Chile, que proporcionó los medicamentos (Prednisona e Isoniazida) usados en este estudio.

\section{REFERENCLAS}

1 Dubouts, Victor. "Muscle disorders in childhood". Saunders, 1978, pp. 19-69.

2 Inutn M. Siegel, M.D. "Muscular dystrophy". Postgraduate Medicine, Yol. 69, N.22, Feb. 1981.

3 Douber, $R$. $M$."Treatment of musculax dystrophy with steroids". New England J. of Med. 268: 912, 1963.

4 "Ineffective treatment of muscular dystrophy with an anabolic steroid and other measures". New England J. of Med 278: $475,1965$.

5 Leon Charush, M.D. "Anabolic steroids in the management of muscular dystrophy". Pediatrics, Vol. 36, N. ${ }^{\circ}$ 3: 402 . Part 1. Sept 1965.

6 D. B. Drach, KV. Toykn, E Myer. "Prednisone in Duchenne muscular dystropby". Lancet, December 14, 1974, p. 1409.

7 Henry Kendal' and Flonence Kendall." "Muscles testing and function". The Willians and Wilkins Company, 1949. Ed.

8 V. Dubourtz and J. Heakmat. "Management of muscular dystrophy". British Medical Bulletin. Vol. 36, N.², p. 142, May 1980.

9 Kent G. Allsop, Fined A. Zher. "Lose of strength and functional decline in Duchenne's distrophy". Archives of Neuralogy. Vol. 38, July 1981 , pp. 406-411.

10 Inetn $M$ Siegel, J. E. Miller, at al "Failure of corticosteroid in the treatment of Duchenne muscular dystrophy". Illinois Medical Joumal 145: 32-33, January 1974.

II Munsat, T. L. a af. "Serum enzime alterations in neuromuscular disonders". Jarra 226: 1536, 1973.

12 Theodore L. Muract ard Water. "Serum CPK and Prednisone treated muscle weakmess". Neurology 27: 96-97, January 1977.

13 Takahashi, K.; Oimomi, M., at al "Response of senum creatine phosphokinase to steroid homnone". Archives of Neurolagy 32: 82-91, Feb. 1975.

14 Technical Bulletin N. ${ }^{\circ} 661$. Sigma Chemical Company, 1978. 3500 DE Kald Street Saint Lowis MO. 63118, USA. 